\title{
BUILDING BRIDGES, MINDING THE GAPS: INVOLVING THE PERSPECTIVES OF OLDER PEOPLE IN CREATING RESILIENT HEALTHCARE INFRASTRUCTURE TO DISASTERS
}

\author{
Yvonne Merino ${ }^{1}$ \\ Andrea Vásquez ${ }^{2}$ \\ Katitza Marinkovic ${ }^{3}$ \\ 1) Pontifical University Catholic of Chile, Chile \\ 2) University of Edinburgh, Scotland \\ 3) University of Melbourne, Australia
}

\begin{abstract}
Introduction: Natural disasters have increased over the last decades. As the world population is also ageing, older people have become more exposed to disasters, with the United Nations highlighting the critical role of healthcare infrastructure in disaster contexts. Traditionally, research on disaster resilience has focused mainly on disasters' negative effects on older people as a vulnerable group, and there is scarce research in engineering that incorporates older people's needs and experiences as a group that is also capable and resilient. This project responds to the lack of research that incorporates the assessment of resilience in healthcare facilities that incorporates the perspectives of older people as its final users.
\end{abstract}

Objective: This paper presents a methodological approach to integrating the physical assessment of resilience in healthcare facilities with the perspectives of older people. It corresponds to a study still being conducted in the central zone of Chile. This region is regularly exposed to earthquakes and concentrates the largest number of complex healthcare facilities in the nation, targeting around $40 \%$ of its older population.

Methods: A collaboration between two $\mathrm{PhD}$ research projects is assessing healthcare facilities' experience of large earthquakes and the relationships between facilities in disaster contexts and the perspectives of older people. This is a mixed-methods study, that will combine a qualitative approach (semi-structured interviews and focus groups) with seismic engineering resilience modelling.

Results: Preliminary results helped identify earthquakes' potential effects on functionality from the perspectives of older people and the relationships between healthcare facilities. This study can contribute to healthcare facilities' earthquake resilience by offering a broader perspective on the links between older people's disaster experiences and healthcare facilities.

Keywords: earthquake resilience, healthcare facilities, older people.

(C) 2019 Merino, Y. Vásquez, A, Marinkovic, K. This is an Open Access article distributed under the terms of the Creative Commons Attribution-NonCommercial 4.0 International License (http://creativecommons.org/licenses/by-nc/4.0), permitting all non-commercial use, distribution, and reproduction in any medium, provided the original work is properly cited.

ISBN: 978-91-88898-41-8

DOI: https://doi.org/10.15626/rea8.06 


\section{CONTEXT AND BACKGROUND}

\subsection{Disasters: their impact in healthcare infrastructure and older people}

Disasters are increasing worldwide and earthquakes disrupt the normal functioning of critical infrastructure such as healthcare centres. At the same time, the world population is ageing at a faster rate. By 2050 it is expected that people aged 60 and more will represent a quarter of the world's population [1]. This means that more older people will be exposed to disasters. A large part of the literature on this topic has identified older people as a vulnerable group due to their higher levels of chronic illness and need for external support during disasters [2]. Much of this support is provided by healthcare services. As proposed by the Sendai Framework for Disaster Risk Reduction (SFDRR) 2015 - 2030 [3], healthcare services are critical infrastructure during the immediate aftermath of a disaster. In addition to medical attention, they provide other important types of support for vulnerable groups. In line with this, the SFDRR states that one of the pillars for building more resilient communities is including the experiences and knowledge of those who are more vulnerable to disasters.

\subsection{Case study, definitions and purpose of this research}

In this section, we'll describe the conceptual framework used to propose an integrated methodological approach to communities' resilience. We'll also discuss some of the gaps in the literature that drove us to study older people's response to disasters in the healthcare infrastructure as an example of critical systems.

\section{Resilience: towards an integrative approach between infrastructure and communities}

Resilience is a concept widely explored across different disciplines. In this paper, we focus on seismic resilience, understood as the capacity of a spatially distributed social unit to build preparedness for risk mitigation, resist the effects of disruptive earthquakes, and recover functionality as rapidly as possible through strategies that minimize loss [4]. A resilient system can be analyzed using different indicators, and at the scale of communities exposed to earthquakes, resilience should be studied including social, economic, institutional and infrastructure aspects with a focus on community capital [5].

In this context, healthcare infrastructure capacity represents one of the main components for a resilient community. As a critical system, it's expected to have continued operability after an extreme earthquake to allow the delivery of medical assistance and the supply of basics needs for the people dealing with health issues [6]. Great earthquakes have affected the functionality of healthcare systems worldwide; for example, Illapel and Maule in Chile [7, 8] and Japan [9]. Determination of post-earthquake effects on communities have evidenced the relevance of preparedness for resilience, with prepared people showing an enhanced capacity to respond to disasters. Even more, the literature has suggested that older people are willing to share their past experiences on disasters and recognise themselves as assets in disaster preparedness. However, their role appears limited mostly to the family and community levels $[10]$. 
A large part of the literature explores older people's vulnerability to disasters, because of their tendency to depend more on external resources to cope with the aftermath of disasters. Some characteristics that make them more frail to disasters are chronic diseases, lack of economic resources and low mobility $[2,11]$. Nevertheless, even though older people are more vulnerable to disasters, there is still a lack of evidence about older people's past experiences with these events. Few studies have explored older people's perspectives on disasters, their lessons learned and the support needed by them to face them. This knowledge can be key for disaster preparedness [3], but few studies have explored how older people's learned lessons could be incorporated to a formal disaster response. Although the literature has described how healthcare services play a critical role responding after an event, scarce studies have explored the connections between the resilience of healthcare services and the perspectives of older people who have experienced disasters in the past.

\section{Minding the gap: conceptual framework of this study}

The seismic resilience of healthcare infrastructures has been described in terms of four dimensions: (i) robustness and (ii) redundancy, referred to the physical capacity of the system, and (iii) rapidity and (iv) resourcefulness, referring to a system's capacity in terms of functionality (i.e. human and physical resources) [12].

Based on these dimensions, this study understands the contribution of older people to the recovery of healthcare systems (i.e. a healthcare service) in the following way

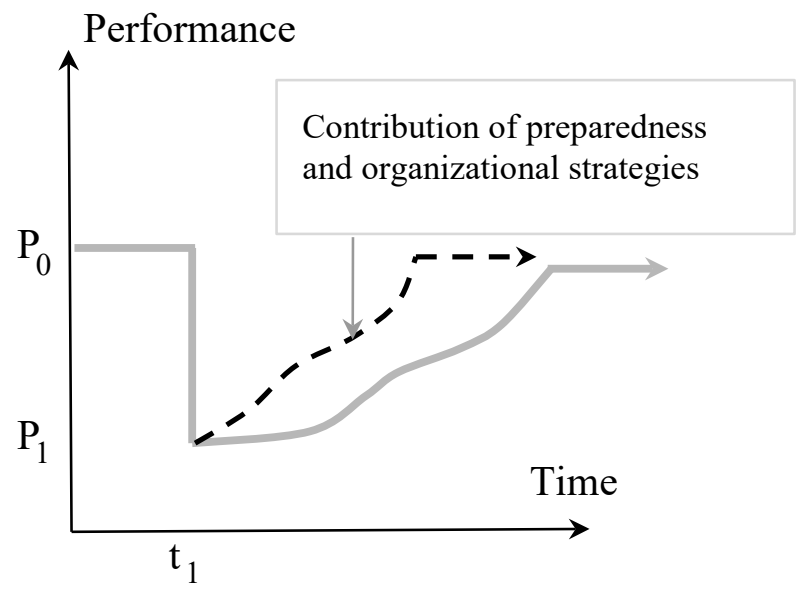

Figure 1. Robustness and rapidity in resilience.

Robustness and rapidity are shown as a curve in Figure 1, with performance (vertical axis) and time (horizontal axis) as the main variables to measure the rapidity dimension of resilience. The gray continuous line tracks the performance of the system across time, expected to reduce from $\mathrm{p} 0$ to $\mathrm{p} 1$ due to the initial loss associated to the event occurrence.

After $\mathrm{t} 1$, the recovery process is a function of several variables including: preparedness, societal response and the organizational capacity to mobilize the available resources and human capital. Accordingly, response and recovery actions from the community are as critical as the system's physical capacity. The integrated responses of human groups tactically managing resources provide the biggest contribution to enhanced resilience, understood as decreasing the time needed to reach a desired performance level [13]. Due to the multiple variables involved, recovery curves are usually considered from assumptions or meta models, making them 
difficult to measure or predict. However, there is empirical evidence that community preparedness results in shorter recovery time. After the Mw8.2 Pisagua earthquake in Chile, the resilience of the healthcare system showed a proper response that was the result of united actions anticipated in the emergency plans of healthcare facilities, which was superior to their individual capacities [14].

According to Cimellaro et al [13], the recovery process in the healthcare system depends on the different recovery paths and timing of the multiple resources and organizational networks within the system. In this context, if a priority group has an enhanced recovery as a result of a comprehensive preparedness and an anticipated seismic response, the resilience of the healthcare system (e.g. healthcare infrastructure) should increase. Thus, this study has the hypothesis that, after $\mathrm{t} 1$, incorporating older people's lessons from past disasters can have a positive impact by contributing to the rapidity of the healthcare service's recovery. Thus, their knowledge could improve the preparedness of healthcare services for future events. Our second hypothesis is that older people can have a positive impact on the response and performance of healthcare services (e.g. helping other older people to evacuate). A prompt response is crucial to reduce damages in the aftermaths of disasters.

\section{Older people and their previous experiences of disasters}

Most of the available literature on older people in disasters has focused on describing their vulnerability based on their socio-demographic characteristics (i.e. age, socioeconomic status, gender), chronic diseases, low mobility and lack of resources to deal with the immediate aftermath of a disaster $[2,11]$.

Defining older people as a vulnerable group due to their healthcare needs (i.e. chronic diseases) has made them a priority for healthcare infrastructures [3]. Assuming that older people depend on healthcare services has impacted how we understand healthcare infrastructure as critical for them mostly in terms of their need for healthcare. For example, the United Nations emphasised the critical role of functioning of healthcare services to cope with the aftermaths of a disaster, especially for vulnerable groups like older people. Nevertheless, scarce studies have explored the perspectives of older people with respect to healthcare services in terms of the support they can provide and how they identify as contributors to the resilience of the healthcare services in the context of a disaster.

In this context, Sendai Framework 2015-2030 [3] has suggested that to promote effective disaster preparedness, we need to include the perspectives of the people affected by disasters. The perspectives of older people in disasters are critical to explore lessons learned in past events, and this knowledge should be shared with younger generations. Even though there is scarce literature on the perspectives of older people in disasters and their past experiences $[10,15]$, some studies have found that older people consider themselves important actors for preparedness due to their experience. Their lessons learned from past events are mostly transmitted within their families and at the community level, but there is a gap in how that knowledge is translated to the formal response [10] carried out by institutions such as healthcare services.

In summary, even though there is evidence describing older people as vulnerable to disasters, there is an emergent recognition of them as contributors to disasters preparedness. However, it is still unclear how to transfer their knowledge beyond their families and communities. 


\section{Purpose of this study}

This paper presents an interdisciplinary methodological approach to integrate older people' perspectives and past experiences in disasters with the assessment of resilience in healthcare infrastructure, with a focus on the preparedness and recovery phases of resilience.

Our hypothesis is that if healthcare systems incorporate the perspectives of older people, this could contribute to anticipate the system's response to disruptive earthquakes and enhance the seismic resilience of healthcare infrastructure for this priority group. Older people can contribute to the recovery phase of resilience in the following ways: (i) during the response of healthcare services, by helping others in case a healthcare building needs to be evacuated and (ii) during the preparedness stage, by using their lessons learned from previous disasters to anticipate challenges that could potentially emerge in a future event.

Thus, an engineering perspective on the assessment of resilience in healthcare infrastructure could be integrated with older people's perspectives and contributions to gain a better understanding of the recovery phase of healthcare infrastructures. Older people can have a positive impact in healthcare services' prompt response and the resilience of healthcare infrastructure

\section{METHODS: TOWARDS AN INTERDISCIPLINARY APPROACH}

This study was based on a bottom-up approach, meaning that it addresses community needs as well as the theoretical gap on how to involve older people's perspectives on the assessment of healthcare infrastructure resilience.

Previous studies conducted by members of this research team explored the assessment of resilience in communities (survivors of the 1960 earthquake [16]) and the healthcare network. The second study used mixed methods to understand the performance of primary healthcare services after an earthquake. The team carried out interviews with key stakeholders (i.e. heads and emergency teams) from primary healthcare services and a hospital to learn about the challenges they faced during the Pisagua earthquake and how they solved them [14]. Its findings supported older people's critical role during disaster response and how it was closely related to their arrival at healthcare services immediately after the earthquake.

\subsection{Case study}

The administration and operation of the public health system in Chile is geographically divided into different healthcare services. Each healthcare service is in charge of medical facilities in multiple provinces. This study focuses on the six healthcare services in the central zone of Chile that concentrate $40 \%$ of the total population of the country (mainly living in the Metropolitan Region). We considered their demographic profile to identify what healthcare centers had the largest older population exposed to disasters. In the Metropolitan Region, people over 60 years old represent around $20 \%$ of inhabitants, most of them concentrated in the East Healthcare Service. Data was collected at the Healthcare Reference Center (CRS Cordillera) in the East Healthcare Service. This center is located near the San Ramón seismic fault, known for its destructive potential in case of an earthquake [17]. 
The healthcare infrastructure network in Chile is built based on three different assistance levels: primary, secondary and tertiary. The CRS Cordillera is a second level facility, with capacity for outpatient care and specialized medical services. It's receives referrals from the primary assistance level that doesn't offer specialized services nor operating rooms. In turn, it refers critical patients to the tertiary level, which offers specialized hospital services and has the capacity for inpatient care. As part of its organizational structure, the CRS is conformed by a consultative council in charge of transmitting the community's healthcare concerns to the healthcare centre. All the members of the consultative council are older people $(n=13)$

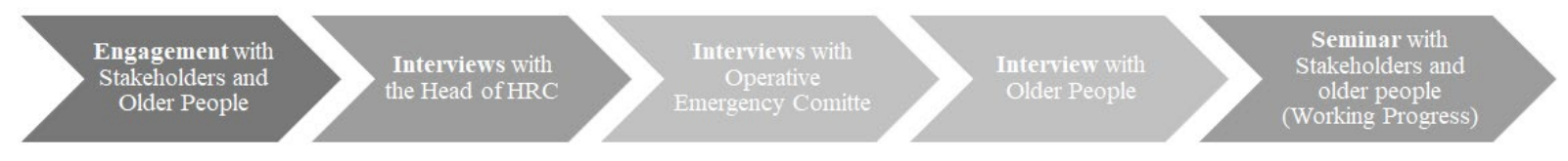

Figure 2. Methodological phases for our proposed integrated approach.

As shown in the Figure 2, our methodological approach consists of five stages. Phase I consisted of approaching and engaging with CRS Cordillera. This stage was critical for the project, as CRS staff work with the consultative council, giving access to older people. The engagement stage was conducted from 2017 until 2019, across different projects previously carried out with the healthcare service.

Phase II consisted on interviewing the head of the healthcare service on its perceptions and concerns regarding older people and disasters. The interview was analysed using thematic analysis, with a focus on the different concerns raised. Phases III and IV are still under progress. During phase III, we will interview the emergency team on their perspectives about the center's functioning after an extreme event and the support provided by older people. During Phase IV, we will perform focus groups with older people from the consultative council. Engaging with the center during Phase I allowed the head of the healthcare centre to schedule meetings in late May 2019.

The final stage of this project will consist on sharing the perspectives of older people and healthcare centre staff during an event aimed at the community. The aim will be for the different stakeholders to dialogue on how to integrate their perspectives to preparedness efforts at the healthcare centre.

\section{RESULTS AND DISCUSSION}

Exploring the perspectives of healthcare service staff and older people who use this service evidenced concerns regarding the following issues: (i) older people's spatial concentration in specific neighborhoods, (ii) ensuring access to aged people with mobility issues (i.e. low mobility) and (iii) how older people could contribute with knowledge from previous disasters to identify potential challenges in case of disaster. Hence, the discussion of the results will follow the three aspects previously described:

\subsection{Spatial concentration of older people at the neighborhood level}

Even though older people have been described as a priority group for healthcare services, their spatial distribution at neighborhood level needs to be explored in more detail. Given the 
heterogeneity of neighborhoods in the central zone of Chile and the areas in this study, it is important to know how older people are distributed. From the CRS's point of view, this may impact their use of the healthcare network, as older people usually attend the healthcare service more frequently than other age groups.

According to Sendai framework [3], healthcare infrastructure is critical for communities, especially during the first response to disasters and for older people. Previous studies on healthcare response to disasters have shown that older people go to healthcare services to look for different types of support that go beyond medical attention [14]. This leads to a higher inflow of older people to healthcare centers and an over demand in certain services.

However, it's not very clear what healthcare services in the network are the most impacted. A few studies [14, 18, 19] have suggested that primary healthcare services are more impacted by this behaviour. On the other hand, it's important to consider the vulnerabilities of the aged population, as neighborhoods with more older people will see these everyday challenges intensified in disaster contexts: low mobility, the need for external resources to cope with the disaster aftermaths and healthcare issues (i.e. chronic illness). For example, in Chile, more than $60 \%$ of older people report having at least one chronic disease such as hypertension [20].

Thus, to create a resilient healthcare infrastructure, we need to consider how healthcare centres interact with the demographic characteristics of their surroundings. These characteristics influence the population they will potentially serve and the epidemiological issues involved, with important implications for evacuation in case of an extreme events. The following section focuses on this topic.

\subsection{Healthcare infrastructure accessible for older people}

One of the concerns shared by the healthcare service's stakeholders is that older people may have mobility issues that may require the use of wheelchairs and longer times to when using stairs, demanding more lifts and assistance from the healthcare service. This has profound implications for evacuation plans: having an increasing aged population may result in longer evacuation times at the healthcare center. At the CRS Cordillera, the evacuation route goes from the second floor to the safety zone in the first floor and involves stairs. This means that people with mobility problems will need support from others to evacuate.

As suggested by recent literature, older people are a heterogeneous group, with some facing mobility issues and others with the capacity to help others evacuate. The latter can be an important resource for healthcare services to achieve shorter evacuation times.

\subsection{Older people as a resource of knowledge}

In addition to the previous issues, the contribution of the older people to an enhanced resilience based on the past experiences goes towards the direction of the four phases of disaster 
management proposed by FEMA (i.e. Mitigation, Preparedness, Response and Recovery), as the community actions and knowledge are involved in all of them, not as the vulnerable components of the system, but as the source of knowledge needed to create strategies to minimize the impacts of hazards, as mitigation is declared the "cornerstone of disaster management".

From an engineering perspective, the interdisciplinary approach presented in this article is an example of complementing different practices with earthquake engineering to help create more resilient communities. The societal and institutional responses of critical systems, such as healthcare facilities, are critical to enhance seismic resilience in communities [4]. They need to be incorporated as a fundamental part of resilience analysis, as they provide crucial information regarding a system's recovery across time. This variable is highly difficult to measure when assessing infrastructure resilience, and is usually estimated by engineers based on data from previous events. In this way, second-generation performance-based earthquake-engineering focuses on seismic risk and functionality loss analysis. Meanwhile, the qualitative exploration of human capital and people's perspectives can contribute to determine recovery paths across time, as well as the variables help improve it in healthcare infrastructure serving high numbers of older people.

\section{CONCLUSIONS}

Our findings from this case study partially support our hypothesis: the healthcare service considered that including older people's perspectives was important to be better prepared for future disaster events. However, it's important to consider that this project is still being implemented and data collection with older people has not begun yet.

Older people were found to be important contributors for disaster preparedness and recovery. This is due to lessons from past disaster experiences that help them anticipate future challenges. Older people can be contributors in the immediate aftermath of a disaster and support other older people in need of assistance, especially during an evacuation process. The involvement of older people in the preparedness and recovery phases of resilience is especially important in contexts where the population is ageing fast and has a high level of disaster exposure.

Summarising, an integrative approach to resilience can benefit from integrating both perspectives: the analysis of physical infrastructure and its interactions with priority populations such as older people. An holistic approach can provide a valuable input and improve the assessment of resilience in healthcare infrastructure.

Future research is needed to explore how to measure the impact of involving the perspectives of the elders on the resilience of healthcare infrastructure. One of the challenges for this project was that it depended on the health centers' active engagement to share its concerns and for the research team to incorporate this information with data from the center's territorial context in terms of the distribution of older people. Finally, for future studies it would be remarkable to address research questions around the possibility of estimating recovery functions for resilience quantification based on the interaction with the population with past experiences in great disasters, as the current literature lacks of such information, and uses few empirical curves from recorded disruptive events. 


\section{ACKNOWLEDGMENTS}

This project is funded by UNIVERSITAS21 network, within the U21's Graduate Collaborative Research Awards 2018, and supported by the National Center for Integrated Natural Disaster Management (CIGIDEN) CONICYT/FONDAP/15110017. The PhD students would also like to thank CONICYT for providing doctoral thesis grants.

\section{REFERENCES}

[1] WHO (s.f.). Envejecimiento y ciclo de vida.

[2] Fernández, Lauren S., et al. "Frail elderly as disaster victims: emergency management strategies." Prehospital and disaster medicine 17.2 (2002): 67-74.

https://doi.org/10.1017/S1049023X00000200

[3] United Nations. Sendai framework 2015-2030

[4] Bruneau et. al (2003). A framework to quantitatively assess and enhance the seismic resilience of communities. Earthquake Spectra. v19 (4). Earthquake Engineering Research Institute (EERI). pp 733-752

[5] Cutter et al (2010). Disaster Resilience Indicators for Benchmarking Baseline Conditions. Journal of Homeland Security and Emergency Management. V 7 (No.51) Issue 1.

[6] Cimellaro et al (2016). Urban resilience for Emergency Response and Recovery: Fundamental concepts and applications. Geotechnical, Geological and Earthquake Engineering, v41. Springer International Publishing Switzerland

[7] Favier, P., Rivera, F., Poulos, A., Vásquez, J., de la Llera, J., \& Mitrani-Reiser, J. (2017). Impact on Chilean hospitals following the 2015 Illapel earthquake. In 16th World Conference on Earthquake, 9-13 January 2017, Santiago, Chile (pp. 1-12).

[8] Kirsch, T. D., et al (2010). Impact on hospital functions following the 2010 Chilean earthquake. Disaster medicine and public health preparedness, 4(2), 122128. https://doi.org/10.1001/dmphp.4.2.122

[9] Nohara, M. (2011). Impact of the Great East Japan Earthquake and tsunami on health, medical care and public health systems in Iwate Prefecture, Japan, 2011. Western Pac Surveill Response J, 2(4), 24-30.

[10] Howard, A., Blakemore, T., \& Bevis, M. (2017). Older people as assets in disaster preparedness, response and recovery: lessons from regional Australia. Ageing \& Society, 37(3), 517-536. https://doi.org/10.1017/S0144686X15001270 
[11] Aldrich, N., \& Benson, W. F. (2008). Disaster Preparedness and the Chronic Disease Needs of Vulnerable Older Adults. Preventing Chronic Disease: Public Health Research, Practice, and Policy, 5(1), 1-7.

[12] Bruneau and Reinhorn (2007). Exploring the concept of seismic resilience for acute care facilities. Earthquake spectra. v23 (1). Earthquake Engineering Research Institute (EERI). pp 41-62

[13] Cimellaro et al (2010). Seismic resilience of a hospital system. Structure and Infrastructure Engineering. v6 (1-2). pp 127-144 https://doi.org/10.1080/15732470802663847

[14] Vásquez et al (2017). Healthcare network's response and resilience in Iquique after the 2014, Pisagua earthquake. 16th WCEE. Santiago, Chile

[15] Brockie, L., \& Miller, E. (2017). Older adults' disaster lifecycle experience of the 2011 and 2013 Queensland floods. International journal of disaster risk reduction, 22, 211-218. https://doi.org/10.1016/j.ijdrr.2016.08.001

[16] Vásquez, A; Repetto, P; Ariztía, M; Ramis, S. (2016). Alertas naturales y preparación ante desastres naturales: Estudio con sobrevivientes de los terremotos y tsunamis de Valdivia en 1960 y del Maule en 2010. III Encuentro Científico Nacional Reducción de Riesgo de Desastres REDULAC.

[17] Armijo, R., Rauld, R., Thiele, R., Vargas, G., Campos, J., Lacassin, R., \& Kausel, E. (2010). The West Andean thrust, the San Ramon fault, and the seismic hazard for Santiago, Chile. Tectonics, 29(2).

[18] Quarantelli, E. L. (1985). Needed Innovation In Emergency Medical Services In Disasters Of The Future

[19] Van Minh, H., Anh, T. T., Rocklöv, J., Giang, K. B., Trang, L. Q., Sahlen, K. G., ... \& Weinehall, L. (2014). Primary healthcare system capacities for responding to storm and floodrelated health problems: a case study from a rural district in central Vietnam. Global health action, 7(1), 23007. https://doi.org/10.3402/gha.v7.23007

[20] PUC, Senama, Caja Los Andes. (2016). Chile y sus mayores. Encuesta calidad de vida 2016. 